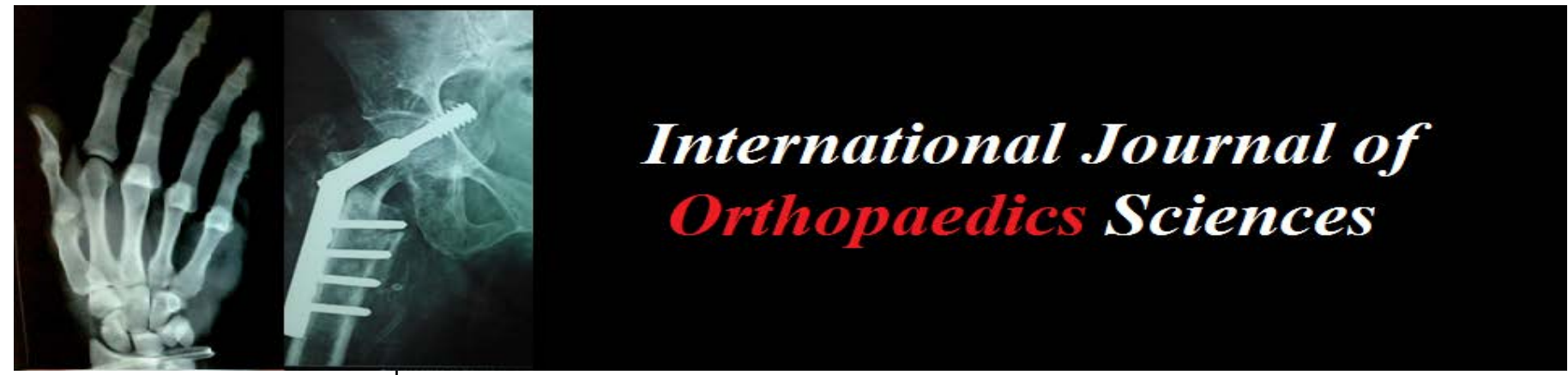

E-ISSN: 2395-1958

P-ISSN: 2706-6630

IJOS 2020; 6(4): 171-172

(C) 2020 IJOS

www.orthopaper.com

Received: 06-08-2020

Accepted: 08-09-2020

\section{Christian Konrads}

${ }^{1}$ Department for Trauma and

Reconstructive Surgery, BG

Klinik, University of Tübingen,

Tübingen, Germany

${ }^{2}$ Department of Orthopaedics, University of Würzburg,

Würzburg, Germany

\section{Patella height assessment in total knee Arthroplasty: Simple adaptation of the Caton-Deschamps index might be sufficient}

\section{Christian Konrads}

DOI: https://doi.org/10.22271/ortho.2020.v6.i4c.2337

Abstract

Multiple patella height indices exist. For application in Arthroplasty detection of not only true patella infera but also pseudo patella infera seems to be important. A modification to the original CatonDeschamps Index could maintain its principles and detect pseudo patella infera in knee Arthroplasty.

Keywords: Patella infera, Patella Baja, Patella Alta, Patella Norma, Knee joint replacement, TKA

\section{Introduction}

In 2016, Caton et al. ${ }^{[1]}$ published the modified Caton-Deschamps Index (mCDI) for knee Arthroplasty. I wondered why the authors did not simply modify the original CatonDeschamps index ${ }^{[2]}$ (CDI) published in 1982 in that way that the new tibial reference point would be the anterior proximal point of the inlay (Fig. 1 b).
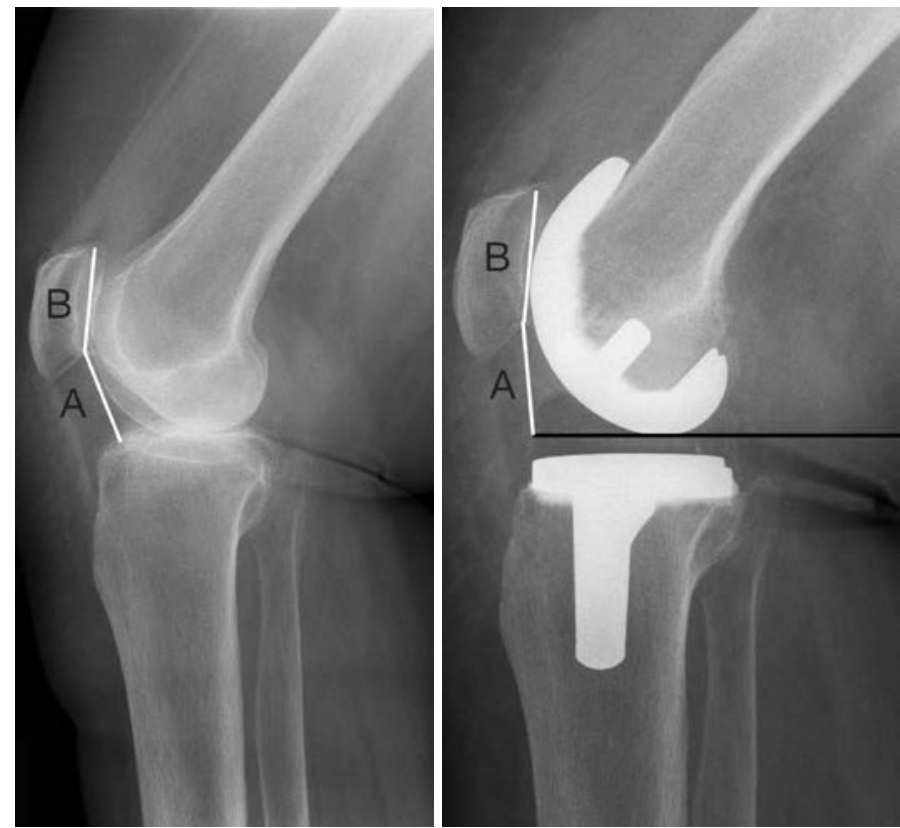

Fig 1a and b: Caton-Deschamps Index (CDI) for native knees (a) and derived Caton-Deschamps Index (dCDI) for Arthroplasty (b)

${ }^{1}$ Department for Trauma and Reconstructive Surgery, BG

Klinik, University of Tübingen,

Tübingen, Germany

${ }^{2}$ Department of Orthopaedics, University of Würzburg,

Würzburg, Germany
$C D I$ Caton-Deschamps Index, $d C D I$ derived Caton-Deschamps Index

This would be an easy modification to the original index conserving the principles in a way that functional patellar height is fully detected. Instead, the published modified CatonDeschamps Index (mCDI) in the paper cannot detect pseudo patella infera (PPI) ${ }^{[3]}$. So, it is 
merely an alternative to the Insall-Salvati index (ISI) ${ }^{[4]}$. A PPI due to an inlay exchange to a thicker one would remain undetected by both the mCDI and the ISI.

The modification I propose (Fig. 1) can be found in published literature, but to my best knowledge it has not been fully analyzed regarding inter- and intraobserver agreement and validity ${ }^{[5]}$. I would call it "derived Caton-Deschamps Index" (dCDI) (Fig. 1 b). Strong advantages of the dCDI are that the reliability is probably similar to that of the original CDI and the reference range should be identical.

For full assessment of all components of patella infera, namely true patella infera (TPI) and pseudo patella infera (PPI), a combination of the original CDI preoperatively and the dCDI postoperatively and the ISI (pre- and postoperatively) would be sufficient to detect (1) the full amount of patella infera, (2) TPI, and (3) PPI.

Funding: None

\section{Conflict of Interest: None}

\section{References}

1. Caton JH, Prudhon JL, Aslanian T, Verdier R. Patellar height assessment in total knee Arthroplasty: A new method, Int Orthop. 2016;40:2527-2531.

2. Caton J, Deschamps G, Chambat P, Lerat JL, Dejour H. [Patella infera. Apropos of 128 cases], Rev Chir Orthop Reparatrice Appar Mot. 1982;68:317-325.

3. Grelsamer RP. Patella Baja after total knee arthroplasty: is it really patella baja? J Arthroplasty. 2002;17:66-69.

4. Insall J, Salvati E. Patella position in the normal knee joint Radiology. 1971;101:101-104.

5. Xu B, Xu WX, Lu D, Sheng HF, Xu XW, Ding WG. Application of different patella height indices in patients undergoing total knee Arthroplasty, J Orthop Surg Res. 2017;12:191. 